\title{
The relationship between gelotophobia, shame, and humiliation
}

\author{
Judit Boda-Ujlaky \\ University of Physical Education, Budapest, Hungary \\ ujlaky@tf.hu \\ László Séra \\ University of Pécs, Institute of Psychology, Hungary \\ serlasz43@gmail.com
}

\begin{abstract}
Gelotophobia (fear of being laughed at), when it comes to the social context, is usually investigated in the context of bullying. Other studies of gelotophobia show that it can be characterised by the emotion of shame. Shame rarely occurs as a clear, distinct emotion (Scheff 2003), i.e. it is difficult to tell whether the given intensity means shame or another emotion to a person. According to Scheff (2003) there is a shame-spectrum, at one end of which there is the feeling of embarrassment, on the other we find humiliation. In our study we investigated the relationship between self-reported gelotophobia, shame, and humiliation (PhoPhiKat-45, TOSCA-3, Humiliation Inventory). Gelotophobia showed a stronger positive correlation with shame than with cumulative humiliation or fear of humiliation ( $r=0.659$ and $r=0.332$ and $r=0.355)$. After partialling out items referring to being laughed at or ridiculed, humiliation proved to be uncorrelated with gelotophobia, whereas with shame it still shows a strong positive relationship (shame: $r=0.568$; cumulative humiliation: $r=-0.001$; fear of humiliation: $r=0.156$ ). Linear regression also confirmed the predictive effect of shame on gelotophobia, but humiliation was not predictive. To conclude, gelotophobia is connected to humiliation only when humiliation contains derision, but other types of humiliation experiences seem to be unrelated.
\end{abstract}

Keywords: gelotophobia, shame, humiliation. 


\section{Introduction}

Leeming and Boyle (2004) argue that shame is experienced in particular social contexts; in other words, a stigmatising discourse is salient in specific interpersonal situations. The experience of becoming the object of derision is one such particular context. While most of us dislike being laughed at in general, some people actually produce extreme reactions in such situations - these people are referred to as gelotophobes.

The term 'gelotophobia' (derived from gelos, the Greek word for laughter, and phobos, the Greek word for laughter and fear) was introduced by Michael Titze in 1996. He drew on his clinical observations to associate gelotophobia with the Pinocchio Complex (Sellschopp-Rüppel \& von Rad 1977). The most typical symptom of the condition is the fear of being laughed at or being ridiculed, which produces dramatic consequences as far as the sufferers' interpersonal relationships are concerned (Ruch 2009: 2). Today, gelotophobia is not necessarily associated any longer with a pathologic degree of fear as originally described by Titze and indeed it has also been described and interpreted as a personality trait of normal individuals (Ruch 2009).

In their first major empirical study of gelotophobia, Ruch and Proyer (2008) argued that gelotophobes can be distinguished from shame-based neurotics and non-shame-based neurotics alike as well as from normal controls. At the same time, gelotophobes may be regarded as a subgroup of shame-based neurotics as sufferers of gelotophobia: "They fear to be laughed at because of things they are ashamed of" (Ruch \& Proyer 2008: 64).

Another important research that supports the relationship between shame and gelotophobia was carried out by Platt and Ruch (2009). In two samples of healthy adults (from Germany and the UK) they found that gelotophobes experienced more intensive shame and it took longer for them to get over the feeling than for others. Also, in a typical week, gelotophobes experienced shame more often than non-gelotophobes, while no correlation was found with the speed of onset. At the same time, only the intensity of shame was found to correlate with gelotophobia and no other negative emotions were shown to intensify in the same context. It is, therefore, quite conceivable that a reluctance to feel joy combined with a propensity to shame led to gelotophobes misinterpreting laughter in ambiguous situations (Ruch et al. 2009: 108). We must note here that in individuals who have often experienced threat in some form or another, the concept of threat becomes accessible in the long term and these people become more sensitive to the signs of threat in interpersonal situations. These individuals are more likely to experience ambiguous situations as threatening (Higgins 1989).

A direct relationship between shame and gelotophobia was examined in a study by Proyer, Platt and Ruch (2010). Using TOSCA-3, the authors found that gelotophobia was linked to a proneness to experience shame and a tendency to externalise. They also found that gelotophobes had a more pronounced sense of guilt and showed less pride than either gelotophiles or katagelasticists.

Other emotions that belong to the shame family were not directly investigated; however, some theories may prove to be very helpful in developing a better understanding of the fear of being laughed at or becoming the object of derision. Billig, for instance, draws attention to the basic social function of embarrassment caused by laughter and ridicule (2005). He argues that society would fall apart if we chose to ignore the rules which keep it operational; the members of society must, therefore, persuade each other to act in accordance with those rules and even to internalise them. In his interpretation the feelings of shame and embarrassment, conveyed by laughter and ridicule, serve precisely that purpose. What actually happens is that children who 
are laughed at by their parents learn that those who are embarrassed are to be laughed at. (N.B. Billig himself distinguishes between situations where people tend to experience shame and where they rather feel embarrassed according to the relative severity of the underlying reason[s]). In fact, everybody who is laughed at becomes embarrassed, but gelotophobes react with a more intense form of embarrassment: shame.

The theory of gelotophobia is supported by research by Miller and Tangney (1994). Here, the participants were asked to recall situations in which they experienced embarrassment and/or shame. The researchers found that the embarrassing situations were perceived as more humorous than those when shame was experienced. The main difference between the feeling of embarrassment and that of shame seems to be that an audience is invariably present in the case of the former (Tangney et al. 1996). Similarly, while a moment of embarrassment is not usually found humorous by anyone at first we can laugh at the situation later and we can even learn to make others laugh by drawing their attention to the points which we think of as funny in retrospect. This appears to be working as a coping mechanism as it transforms the pain experienced in the past to the fun of the present moment (Billig 2005; see the theory of gelotophilia). Parrott and Smith (1991, cited by Billig 2005) found that the participants were laughed at in some 40 percent of the descriptions of various embarrassing situations. Speaking of gelotophobia, it is important to mention here that it is not only ridicule that provokes shame in gelotophobia sufferers but indeed all forms of laughter, even the positive forms (Ruch et al. 2009).

At the same time, derision is also an important component of humiliation. In researching their Humiliation Inventory, Hartling and Luchetta (1999) found that the highest factor loads were associated with strong criticism or censure, derision, teasing, ridicule, and exclusion from the group. Based on their findings in one research project, Elison and Harter (2007) point out that "[c]ritical to the experience of humiliation was the role of an audience, peers who typically laughed at the victim" (p. 319, italics in the original). On two other occasions the same authors, aiming to distinguish emotions that belong to the shame family (Elison \& Harter 2007), found that humiliation was an intense emotion produced in reaction to criticism by an ill-intentioned audience (e.g. a glance interpreted as scathing, laughter) after a norm of any kind was broken, where the criticism was congruent with the criticised person's self-image. Shame, on the other hand, was defined as a feeling of similarly high intensity but one which occurred typically when a moral norm was broken and regardless of the presence of an audience and the intentions thereof. Humiliation was also found to be different from shame in that it involved the mortification or downgrading of someone, with the interaction playing a more important part and a negative self-assessment a lesser role. As opposed to humiliation, shame is often experienced as something that one has deserved and as such it may involve a will to change, while humiliation is clearly not adaptive (Klein 1991). In essence, shame is thought to be experienced in situations generated by ourselves in some way or another while humiliation generally occurs in situations created by others. All in all, humiliation can be looked at as a very powerful emotion produced in degrading contexts (i.e. where degradation of the self takes place in the eyes of others). In contrast to that, while also very intensive, shame mainly has moral associations (Elison \& Harter 2007). As a consequence, experience of embarrassment or of humiliation may be moderated by gelotophobia, or, gelotophobia itself might be caused by humiliation. Again, parallel with the phenomenon of gelotophobia, the age of the people involved is not at all insignificant when it comes to experiencing embarrassment or humiliation: e.g. while teenagers' primary reaction to embarrassment is derision, adults are more likely to 
respond with understanding and empathy (for a summary of underlying research, see Miller 2007).

In summary, while research has established that gelotophobia is related to shame, humiliation in turn can be defined as an intensive form of shame. Besides, the emergence of gelotophobia can also be caused by the bullying people may suffer from at school, and indeed bullying may take the form of ridicule. In that connection, Elison and Harter (2007) propose that the humiliation produced by bullying motivates shootings at schools, i.e. humiliation could be what moderates the relationship in question. Still, no research has yet been undertaken into the relationship between humiliation and gelotophobia. Our own research is a cross-sectional pilot study which looks into the most obvious connections between the two phenomena; however, an examination of the interrelatedness of bullying, gelotophobia, humiliation and aggression has been long overdue. Indeed, to be able to lighten the relationship between gelotophobia, humiliation, and bullying, a more detailed and longitudinal research is needed, therefore we do not consider bullying at all here.

\section{Method}

\subsection{Participants}

The sample consisted of $\mathrm{N}=90$ Hungarian-speaking participants (27 male and 63 female) between 18 and 63 years of age $(M=24.50, S D=8.20)$. Education and/or qualifications of the participants: primary or secondary school leaving certificate (1-1), baccalaureate (54), vocational diploma (6), or university degree (28). Research is being carried out in the framework of our enquiry into the experience of shame, sense of guilt, and humiliation among athletes. The sample, therefore, includes a relatively high number of athletes (both amateur and professional; with the professionals performing on various levels and producing various results).

\subsection{Materials and procedure}

The surveys were administered by two university students, with a four-month interval between the two rounds. The respondents $(2 \times 45)$ were mainly the students' own personal acquaintances, and the answers to the questions were simply jotted down by them with pen on paper. The questionnaires, including clear and transparent instructions, were distributed among the participants and collected at a later date. For that reason, we had no way to check if our request that they give quick answers without too much thinking was observed or not.

The package included three questionnaires, some general guidance, a statement of acceptance of the terms of participation, and demographic data.

The first questionnaire was PhoPhiKat-45 (Ruch \& Proyer 2009). It included 3x15 items intended to measure varying degrees of gelotophobia, gelotophilia, and katagelasticism, respectively, with the help of the Likert scale (1=strongly disagree, $4=$ strongly agree). This questionnaire had already been tested on a Hungarian sample (Boda-Ujlaky 2014) and proved to be a reliable instrument.

The second questionnaire was TOSCA-3 (Tangney et al. 2000), a scenario-based measure of shame, detachment/unconcern, externalisation, guilt, and pride (hubristic and achievementoriented). This one had not been used in Hungary before. Therefore, although we followed the established manner of questionnaire translation (i.e. independent linguists first translated the text 
from English to Hungarian; next, the material was translated back from Hungarian to English; and then it was proofread by a third, independent person, who compared the original and the English translation and proposed any modifications necessary), we were not in a position to draw conclusions that could be said to be valid and unambiguous in all respects. This questionnaire included 16 different situations. The participants were asked to respond based on the Likert scale ( $1=$ not likely, $5=$ very likely) in a total of 79 cases.

The third questionnaire was the 'Humiliation Scale' (Hartling \& Luchetta 1999). It contained 32 items, with the first 12 assessing the respondents' humiliation experiences and the remaining 20 seeking to obtain information about their fear of humiliation (again, on the Likert scale: $1=$ strongly disagree, $5=$ strongly agree). As this third questionnaire had not been used in Hungary either, here too we followed the established way of translation (see under the description of the $2^{\text {nd }}$ questionnaire above). In addition, it included open-ended questions concerning any experience of shame, guilt, and/or humiliation in connection with sporting events.

\section{Results}

Of a total number of 90 participants, we found 13 gelotophobes (14.44\%), i.e. twice as many as the Hungarian average we found in different studies. One reason for this could be a lower proportion of the respondents being from the capital - research has repeatedly confirmed a higher concentration of gelotophobes among those living in smaller places in the country (see Platt \& Forabosco 2012). Another reason could be athletes' acute experiences of humiliation (although sportsmen and -women have been found to repress shame responses, see Tracy \& Matsumoto 2008).

Based on the Cronbach-alpha values, PhoPhiKat-45 proved to be a reliable testing instrument with $\dot{\alpha}=0.809, \dot{\alpha}=0.862$, and $\dot{\alpha}=0.825$ with regard to gelotophobia, gelotophilia, and katagelasticism, respectively.

TOSCA-3 did not return the same degree of reliability: shame: $\alpha=0.857$; detachment: $\alpha=0.722$; guilt: $\alpha=0.846$; externalisation: $\dot{\alpha}=0.712$; hubris: $\dot{\alpha}=0.373$; and (achievement-oriented) pride $\alpha=0.595$.

As for the Humiliation Scale, here the Cronbach-alpha values are high, associated with a high degree of reliability (cumulative humiliation: $\alpha=0.886$; fear of humiliation: $\alpha=0.950$ ).

All in all, the results can be interpreted in light of the examination of the relationship of gelotophobia, gelotophilia, katagelasticism, shame, guilt, cumulative humiliation, and fear of humiliation. In seeking to obtain insight into the relationship between shame and humiliation on the one hand and gelotophobia on the other, participants' potential disposition to gelotophilia or katagelasticism was not assessed.

For the purposes of statistical analysis, we used the SPSS 22 software tool and carried out correlation (all the results are shown in Table 1.) and linear regression analyses. We found $1 \%$ significant positive correlation between gelotophobia and shame $(r=0.659)$, confirming the findings of earlier research that revealed a meaningful relationship between them. The same $1 \%$ significant positive correlation was found with cumulative humiliation and with the fear of humiliation ( $r=0.332$ and $r=0.355$, respectively). In other words, shame and humiliation were both found to go hand in hand with gelotophobia, showing strong correlation and a medium degree of correlation, respectively. Both components of the Humiliation Scale, however, originally included two items directly related to derision. As both of these are set to increase the 
extent of correlation, we decided to remove them from the next analysis, which then turned out to confirm a very high a degree of correlation with shame (1\% significant positive correlation, $\mathrm{r}=0.568$ ), while correlation with cumulative humiliation or fear of humiliation could not be observed on either scale $(r=-0.001$ and $r=0.156)$. I.e. unless the element of derision is directly involved, gelotophobia and humiliation show no correlation with each other.

As for the Humiliation Scale items, only four items showed significant correlations with gelotophobia: items 5 and 10 belong to the cumulative humiliation subscale ("Throughout your life how seriously have you felt harmed by being... [5] ...laughed at? [10] ...embarrassed?"), while the other two, items 15 and 25 ("At this point in your life, how much do you fear being... [15]...ridiculed? At this point in your life, how concerned are you about being... [25] ...embarrassed") belong to the fear of humiliation subscale. The correlations are the following: $\mathrm{r}=0.364 ; 0.365 ; 0.303 ; 0.358$. Results show that feeling embarrassed and being laughed at together count for gelotophobia, while other humiliation items, like exclusion, treated like being invisible, cruel criticism, and so on) show no connection with the concept. Counting subscales only for ridicule-items (5, 7 of cumulative humiliation; 15, 20 of fear of humiliation, Cronbachalphas: 0.758 and 0.885 ), experiences with being laughed at show moderate positive correlation $(\mathrm{r}=0.339)$, while fear of being laughed at shows just a tendency $(\mathrm{r}=0.248)$. This latter result is quite surprising, as gelotophobia means fear of being laughed at. The explanation can be an attempt from the person in face-saving, i.e. nobody wants to appear as humourless, therefore an explicit question toward such a problem creates avoidance or suppression, but a more subtle measurement tool, like PhoPhiKat-45, can reveal the problem. At the same time, cumulative humiliation subscale without the ridicule items $(5,7)$ shows a significant correlation $(r=0.323)$ with gelotophobia, and fear or humiliation subscale without the ridicule-items $(15,20)$ show no correlation $(\mathrm{r}=0.224)$. All the results are shown in Table 1. (however, Cronbach-alphas are 0.863; and 0.929). As a consequence, with this measurement method, gelotophobia seems to be related only to past experiences of being laughed at, whereas fear of being humiliated has no connection with it.

Table 1. Correlations of the gelotophobia scores with the different items and subscales of the Humiliation Scale and TOSCA-3. (H1-32: items of the Humiliation Scale; CH: cumulative humiliation; FH: fear of humiliation; $\mathrm{CH} \_\mathrm{R}$ : cumulative humiliation - ridicule-items only; FH_R: fear of humiliation - ridicule-items only; $\mathrm{CH} \_\mathrm{NR}$ : cumulative humiliation - without ridicule-items; FH_RH: fear of humiliation - without ridicule-items; SH: shame; DT: detached; EXT: externalisation; GUI: guilt.)

\begin{tabular}{|c|c|c|c|c|c|c|c|c|c|c|c|}
\hline $\begin{array}{l}\mathbf{H 1} \\
, 277 \\
, 060\end{array}$ & $\begin{array}{l}\mathbf{H 2} \\
, 226 \\
, 127\end{array}$ & $\begin{array}{l}\mathbf{H 3} \\
, 118 \\
, 430\end{array}$ & $\begin{array}{l}\mathbf{H 4} \\
, 209 \\
, 164\end{array}$ & $\begin{array}{l}\text { H5 } \\
, 364^{*} \\
, 012\end{array}$ & $\begin{array}{l}\text { H6 } \\
, 210 \\
, 156\end{array}$ & $\begin{array}{l}\mathbf{H 7} \\
, 246 \\
, 096\end{array}$ & \begin{tabular}{|l|}
$\mathbf{H 8}$ \\
, 096 \\
, 520
\end{tabular} & $\begin{array}{l}\mathbf{H 9} \\
, 137 \\
, 360\end{array}$ & $\begin{array}{l}\mathbf{H 1 0} \\
, 365^{*} \\
, 012\end{array}$ & $\begin{array}{l}\text { H11 } \\
, 157 \\
, 293\end{array}$ & \begin{tabular}{|l|} 
H12 \\
, 273 \\
, 066
\end{tabular} \\
\hline $\begin{array}{l}\mathbf{H 1 3} \\
, 175 \\
, 238\end{array}$ & $\begin{array}{l}\text { H14 } \\
, 137 \\
, 359\end{array}$ & $\begin{array}{l}\mathbf{H 1 5} \\
, 303^{*} \\
, 039\end{array}$ & $\begin{array}{l}\text { H16 } \\
, 119 \\
, 428\end{array}$ & $\begin{array}{l}\mathbf{H 1 7} \\
, 193 \\
, 194\end{array}$ & $\begin{array}{l}\text { H18 } \\
, 232 \\
, 116\end{array}$ & $\begin{array}{l}\text { H19 } \\
, 265 \\
, 071\end{array}$ & $\begin{array}{l}\mathbf{H 2 0} \\
, 165 \\
, 267\end{array}$ & $\begin{array}{l}\mathbf{H 2 1} \\
, 177 \\
, 235\end{array}$ & $\begin{array}{l}\mathbf{H 2 2} \\
, 077 \\
, 606\end{array}$ & $\begin{array}{l}\mathbf{H 2 3} \\
, 167 \\
, 263\end{array}$ & $\begin{array}{r}\mathbf{H 2 4} \\
, 098 \\
, 511\end{array}$ \\
\hline $\begin{array}{l}\mathbf{H 2 5} \\
, 358^{*} \\
, 013\end{array}$ & $\begin{array}{l}\mathbf{H 2 6} \\
, 062 \\
, 678\end{array}$ & $\begin{array}{l}\mathbf{H 2 7} \\
, 008 \\
, 958\end{array}$ & $\begin{array}{l}\mathbf{H 2 8} \\
, 066 \\
, 661\end{array}$ & $\begin{array}{l}\mathbf{H 2 9} \\
, 223 \\
, 131\end{array}$ & $\begin{array}{l}\text { H30 } \\
-, 030 \\
, 844\end{array}$ & $\begin{array}{l}\text { H31 } \\
, 229 \\
, 122\end{array}$ & $\begin{array}{l}\mathbf{H 3 2} \\
, 221 \\
, 136\end{array}$ & $\begin{array}{l}\text { CH } \\
, 332^{* *} \\
, 002\end{array}$ & $\begin{array}{l}\text { FH } \\
, 355^{* *} \\
, 001\end{array}$ & $\begin{array}{l}\text { CH_R } \\
, 339^{*} \\
, 020\end{array}$ & $\begin{array}{l}\text { FH_R } \\
, 248 \\
, 092\end{array}$ \\
\hline $\begin{array}{l}\text { CH_NR } \\
, 323^{*} \\
, 030\end{array}$ & $\begin{array}{l}\text { FH_NR } \\
, 224 \\
, 130\end{array}$ & $\begin{array}{l}\text { SH } \\
, 659^{* *} \\
, 000\end{array}$ & $\begin{array}{l}\text { DT } \\
-, 205 \\
, 057\end{array}$ & $\begin{array}{l}\text { EXT } \\
, 230^{*} \\
, 031\end{array}$ & $\begin{array}{l}\text { GUI } \\
, 138 \\
, 202\end{array}$ & & & & & & \\
\hline
\end{tabular}


Linear regression analysis confirmed these results: while shame $\left(\mathrm{R}^{2}=0.317\right)$ and katagelasticism (to a much lower extent, though) were both found to predict gelotophobia, the other variables were not, showing humiliation to be independent of gelotophobia.

To be able to get a more complex picture on shame, humiliation, and ridicule, we need to examine their interrelations. The correlation between shame and cumulative humiliation is $\mathrm{r}=0.468(\mathrm{p}<0.01)$, fear of humiliation $\mathrm{r}=0.526(\mathrm{p}<0.01)$, thus we find a medium-high positive relation between the concepts. After partialling out items referring to being ridiculed and laughed at, these coefficients prove to be $r=0.197$ and $r=0.424(p<0.01)$, thus, we can detect a relationship only between shame and fear of humiliation. This result can be interpreted as follows: fear of humiliation is a personality-related, shame-based construct (or at least Humiliation Scale measures that), whereas cumulative humiliation is rather an experience-based, interpersonal construct.

\section{Conclusion}

As we have said, our research is a pilot study, which raises a number of issues. One of them is that the methods we used were based solely on correlation analyses, which made them unsuitable for uncovering cause/effect relationships. Another issue can be that the key question that we presented in our theoretical introduction (i.e. how humiliation, thought to play a role in shootings at schools, and acting out by people who become the object of derision and ridicule, are linked to gelotophobia) finally did not get into the focal point of our enquiry. Moreover, as another limitation we can word that though embarrassment and humiliation mark the endpoints of the so called shame-spectrum, we have only investigated humiliation. Further studies need to clarify the differences between the different classes of shame: embarrassment, shame and humiliation, and their relation to fear of being laughed at.

We embarked on a journey to get some initial results regarding the proposed connection between gelotophobia and humiliation, the latter of which had raised our curiosity as an intensive emotion among the elements of the shame-spectrum. We also believed at the outset that we could find evidence of, and shed light on, gelotophobia's likely appearance in interpersonal situations rather than showing up merely as a personality disposition. It seems quite clear from our findings though that humiliation is linked to gelotophobia only by the former's connection with ridicule and derision, and even then their correlation is not very strong, as opposed to gelotophobia's powerful connection with shame. From this result we can draw the consequence that other forms of humiliation do not predict gelotophobia, ridicule-related humiliation does. On the other hand, considering the derision items (cumulative humiliation and fear of humiliation separately), only past experience with laughter shows a correlation with gelotophobia. Interestingly, there is no significant relationship between gelotophobia scores and fear of being laughed at items of the Humiliation Scale. As an explanation we can suppose that although gelotophobia is present in this sample comprising mostly of athletes, they do not explicitly express fear of being laughed at. At the same time, our sample included but a few gelotophobes. It is, therefore, not inconceivable that the dynamics that we found with respect to the total group of participants are different; as it is, in fact, also not inconceivable that a sample of gelotophobes would have yielded a more pronounced correlation with humiliation. From a methodological aspect, experimental manipulation would be desirable and necessary to uncover any underlying cause/effect relationships. On the other hand, experimenting with negative emotions raises a number of ethical issues, which ought to be taken into careful consideration in the planning phase. 
All in all, our research confirmed gelotophobia being a personality- or humour-disposition linked to shame rather than a response or reaction to certain interactional situations. It was found not to be connected to types of humiliation experiences other than the one of becoming the object of derision or ridicule. Moreover, it was shown to be predicted only by shame, not by humiliation - other forms of humiliation do not predict gelotophobia, ridicule-related humiliation does.

\section{References}

Boda-Ujlaky, J. (2014). 'Assessment of the PhoPhiKat-45 Questionnaire in Hungary'. Paper presented at the $26^{\text {th }}$ International Society for Humor Studies (ISHS) Conference, University of Utrecht, Utrecht, 7-11 July.

Billig, M. (2005). Laughter and Ridicule. Towards a Social Critique of Humour. London: Sage.

Elison, J. \& Harter, S. (2007). 'Humiliation: Causes, correlates, and consequences', in Tracy, J.L., Robins, R.W. \& Tangney, J.P. (eds.), The Self-conscious Emotions: Theory and Research, New York: The Guilford Press, pp. 310-329.

Hartling, L. M. \& Luchetta, T. (1999). 'Humiliation: Assessing the impact of derision, degradation, and debasement'. Journal of Primary Prevention 19 (4), pp. 259-278.

Higgins, T. E. (1989). 'Knowledge accessibility and activation: Subjectivity and suffering from unconscious sources', in Uleman, J. S. \& Bargh, J. A. (eds.), Unintended Thought: The Limits of Awareness, Intention and Control. New York: Guilford Press, pp. 75-123.

Klein, D. C. (1991). 'The humiliation dynamic: An overview'. Journal of Primary Prevention 12 (2), pp. 93-121.

Leeming, D. \& Boyle, M. (2004). 'Shame as a social phenomenon: A critical analysis of the concept of dispositional shame'. Psychology and Psychotherapy: Theory, Research and Practice 77(3), pp. 345-369.

Miller, R. S. (2007). 'Is embarrassment a blessing or a curse?', in Tracy, J. L., Robins R. W. \& Tangney, J. P. (eds.), The Self-conscious Emotions: Theory and Research, New York: The Guilford Press, pp. 245-262.

Miller, R. S. \& Tangney, J. P. (1994). 'Differentiating embarrassment and shame'. Journal of Social and Clinical Psychology 13 (3), pp. 273-287.

Platt, T. \& Ruch, W. (2009). 'The emotions of gelotophobes: Shameful, fearful, and joyless?' HUMOR: International Journal of Humor Research 22 (1-2), pp. 91-110.

Platt, T. \& Forabosco, G. (2011). 'Gelotophobia: The fear of being laughed at', in Gremigni, P. (ed.), Humor and Health Promotion, New York: Nova Science Publishers, pp. 229-252.

Proyer, R. T., Platt, T. \& Ruch, W. (2010). 'Self-conscious emotions and ridicule: Shameful gelotophobes and guilt free katagelasticists'. Personality and Individual Differences 49 (1), pp. 54-58.

Ruch, W. (2009). 'Fearing humor? Gelotophobia: The fear of being laughed at. Introduction and overview'. HUMOR: International Journal of Humor Research 22 (1-2), pp. 1-25.

Ruch, W., Altfreder, O. \& Proyer, R. T. (2009). 'How do gelotophobes interpret ambiguous situations? An experimental validation of the concept'. HUMOR: International Journal of Humor Research 22 (1-2), pp. 63-90.

Ruch, W. \& Proyer, R. T. (2008). 'The fear of being laughed at: Individual and group differences in gelotophobia'. HUMOR: International Journal of Humor Research 21 (1), pp. 47-67. 
Ruch, W. \& Proyer, R. T. (2009). 'Extending the study of gelotophobia: On gelotophiles and katagelasticists'. HUMOR: International Journal of Humor Research, 22 (1-2), pp. 183-212.

Scheff, T. J. (2003). 'Shame in self and society'. Symbolic Interaction 26 (2), pp. 239-262.

Sellschopp-Rüppel, A. \& von Rad, M. (1977). 'Pinocchio - a psychosomatic syndrome'. Psychotherapy and Psychosomatics 28 (1-4), pp. 357-360.

Tangney, J. P., Dearing, R., Wagner, P. E. \& Gramzow, R. (2000). The Test of Self-Conscious Affect-3 (TOSCA-3). George Mason University, Fairfax, VA.

Tracy, J. L. \& Matsumoto, D. (2008). 'The spontaneous expression of pride and shame: Evidence for biologically innate nonverbal displays'. PNAS, 105/33, pp. 11655-11660. 